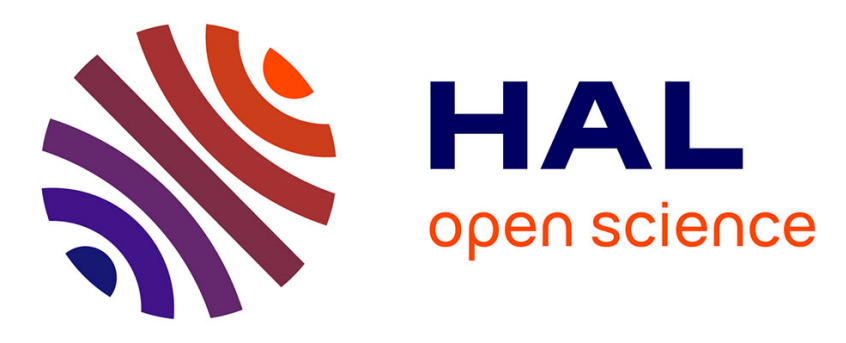

\title{
Scalable Machine Learning for Predicting At-Risk Profiles Upon Hospital Admission
}

Pierre Genevès, Thomas Calmant, Nabil Layaïda, Marion Lepelley, Svetlana Artemova, Jean-Luc Bosson

\section{- To cite this version:}

Pierre Genevès, Thomas Calmant, Nabil Layaïda, Marion Lepelley, Svetlana Artemova, et al.. Scalable Machine Learning for Predicting At-Risk Profiles Upon Hospital Admission. Big Data Research, 2018, 12, pp.23-34. 10.1016/j.bdr.2018.02.004 . hal-01517087v6

\section{HAL Id: hal-01517087 https://hal.inria.fr/hal-01517087v6}

Submitted on 28 Feb 2018

HAL is a multi-disciplinary open access archive for the deposit and dissemination of scientific research documents, whether they are published or not. The documents may come from teaching and research institutions in France or abroad, or from public or private research centers.
L'archive ouverte pluridisciplinaire HAL, est destinée au dépôt et à la diffusion de documents scientifiques de niveau recherche, publiés ou non, émanant des établissements d'enseignement et de recherche français ou étrangers, des laboratoires publics ou privés. 


\title{
Scalable Machine Learning for Predicting At-Risk Profiles Upon Hospital Admission ${ }^{1}$
}

\author{
Pierre Genevès ${ }^{\mathrm{a}, *}$, Thomas Calmant ${ }^{\mathrm{a}}$, Nabil Layaïda ${ }^{\mathrm{a}}$, Marion Lepelley ${ }^{\mathrm{b}}$, \\ Svetlana Artemova ${ }^{\mathrm{b}}$, Jean-Luc Bosson $^{\mathrm{b}}$ \\ ${ }^{a}$ Univ. Grenoble Alpes, CNRS, Grenoble INP, Inria, LIG, F-38000 Grenoble France \\ ${ }^{b}$ Univ. Grenoble Alpes, CNRS, Public Health department CHU Grenoble Alpes, Grenoble \\ INP, TIMC-IMAG, 38000 Grenoble, France
}

\begin{abstract}
We show how the analysis of very large amounts of drug prescription data make it possible to detect, on the day of hospital admission, patients at risk of developing complications during their hospital stay. We explore, for the first time, to which extent volume and variety of big prescription data help in constructing predictive models for the automatic detection of at-risk profiles.

Our methodology is designed to validate our claims that: (1) drug prescription data on the day of admission contain rich information about the patient's situation and perspectives of evolution, and (2) the various perspectives of big medical data (such as veracity, volume, variety) help in extracting this information. We build binary classification models to identify at-risk patient profiles. We use a distributed architecture to ensure scalability of model construction with large volumes of medical records and clinical data.

We report on practical experiments with real data of millions of patients and hundreds of hospitals. We demonstrate how the fine-grained analysis of such big data can improve the detection of at-risk patients, making it possible to construct more accurate predictive models that significantly benefit from volume and variety, while satisfying important criteria to be deployed in hospitals.
\end{abstract}

Keywords: application, big prescription data, volume, variety, experiments

\section{Introduction}

A major challenge in healthcare is the prevention of complications and adverse effects during hospitalization. A complication is an unfavorable evolution or consequence of a disease, a health condition or a therapy; and an adverse

\footnotetext{
${ }^{1}$ This research was partially supported by the ANR project CLEAR (ANR-16-CE25-0010).

* Corresponding author

Email address: pierre.geneves@cnrs.fr (Pierre Genevès)

$U R L:$ pierre.geneves.net (Pierre Genevès)
}

Preprint submitted to Journal of ${ }^{A} T_{E} X$ Templates

February 28, 2018 
effect is an undesired harmful effect resulting from a medication or other intervention. Typical examples include for instance pressure ulcers, hospital-acquired infections (HAI), admissions in Intensive Care Unit (ICU), and death.

From the perspective of complications, healthcare establishements can be considered as risky environments. For instance, in the USA, an estimated $13.5 \%$ of hospitalized Medicare beneficiaries experienced adverse effects during their hospital stays; and an additional $13.5 \%$ experienced temporary harm events during their stays ${ }^{2}$ [1]. However, physician reviewers determined that $44 \%$ of adverse and temporary harm events were clearly or likely preventable [1]. Preventable events are often linked to the lack of patient monitoring and assessment.

One challenging and very interesting goal is to be able to predict the patients' outcomes and tailor the care that certain patients receive if it is believed that they will do poorly without additional intervention. In doing so, hospitals could prevent unnecessary readmissions, adverse events, or other delays in getting well [2]. For instance, if we can precisely identify groups of patients associated with a very high risk of requiring ICU treatment during their stay, then we can optimize their placement as soon as they are admitted, by affecting them e.g. to rooms closer to ICU, thereby drastically reducing transportation delay in life-critical situations in large hospitals. More generally, many complications could be avoided by immediate identification of at-risk patients upon admission and adapted prevention. A crucial prerequisite to any adapted and meaningful prevention is the precise identification of at-risk profiles.

The widespread adoption of Electronic Health Records (EHR) makes it possible to benefit from quality information provided by healthcare professionals [3]. This opens the way for applying AI techniques in building helpful analytics systems for big medical data in which we can have a high level of trust - since drug prescriptions engage the responsibilities of healthcare professionals.

This paper aims to develop an automatic prediction system for identifying at-risk patients, based on a fine-grained analysis of large volumes of electronic health record data. This has long been viewed as a more challenging task than conventional prediction approaches with summary statistics and EHR-based scores $[2,4]$.

\section{Contributions.}

We show how the analysis of very large amounts of drug prescription data make it possible to detect, on the day of hospital admission, patients at risk of developing complications during their hospital stay. We explore, for the first time, to which extent volume and variety of big prescription data help in constructing predictive models for the automatic detection of at-risk profiles. We report on practical experiments with real data of millions of patients and hundreds of hospitals. We demonstrate how the fine-grained analysis of such big data can improve the detection of at-risk patients, making it possible to

\footnotetext{
${ }^{2}$ Temporary harm events are those that require intervention but do not cause lasting harm.
} 
construct more accurate predictive models that significantly benefit from volume and variety, while satisfying important criteria to be deployed in hospitals.

\section{Methodology}

Our methodology is designed to validate our claims that: (1) drug prescription data on the day of admission contain rich information about the patient's situation and perspectives of evolution, and (2) the various perspectives of big medical data (such as veracity, volume, variety) help in extracting this information.

We thus focus on building binary classification models to identify at-risk patient profiles, using distributed supervised machine learning methods. Our approach involves a fully distributed architecture to ensure scalability of model construction with large volumes of medical records and clinical data. The machine learning models that we build yield predictions at hospital admission time.

\subsection{Considered Medical Data and Veracity}

We consider real data from United States Hospitals. Our dataset features more than 33 million discharges from a representative group of 417 hospitals drawn by lot, as provided by the Premier Perspective database, which is the largest hospital clinical and financial database in the United States. Each individual drug prescription engages the responsibility of the prescriber. Each hospital submits quarterly updates of aggregated data. Patient-level data go through 95 quality assurance and data validation checks. Once the data have been validated, patient-level information is available, comprising data consistent with the standard hospital discharge file, demographic and disease state information, and information on all billed services, including date-specific logs of medications, laboratory, diagnostics, and therapeutic services.

The raw data for the year 2006 contains 33048852 admissions, and more than three billion patient charge records, representing $2.8 \mathrm{~Tb}$ of data.

For our study, we focused on basically two kinds of data: (1) population characteristics (age, gender, marital status, etc.) and (2) clinical data including all drug prescriptions (dosage, route of administration of each drug, etc.) for all admissions.

\subsubsection{Filters}

We selected adult and adolescent patients (between 15 and 89 years old ${ }^{3}$ ), hospitalized for more than 3 days. We chose this minimal length of stay of 3 days in order to ensure enough time for manifestation and detection of complications during the stay. Other exclusion criteria for the patients were:

\footnotetext{
${ }^{3}$ We filtered out other ages because this information was biased in the dataset, i.e. age 89 denoting in fact age category $89+$.
} 
- patients hospitalized in surgery, because in surgery medical prescription and its complexity varies considerably according to preoperative, operative and postoperative phase as described in Lepelley et al. [5] and this information was not available in the dataset);

- out-patients and consultations;

- those with no drug prescription at admission; without which we cannot apply our analysis.

These filters retain 1487867 admissions also studied in [5]. We further filter out elective admissions, and finally retain a total of 1271733 eligible admissions.

\subsubsection{Considered Complications and Ground Truth}

To build the complication prediction system, we need labeled data for training and evaluation purposes. We consider four complications:

- death during hospital stay;

- admission to ICU on or after the second day (excluding patients directly admitted to ICU on the first day ${ }^{4}$;

- pressure ulcers that were not present at admission time but developed during the stay;

- hospital-acquired infections developed during the stay.

Labeling a posteriori the occurrence of deaths and admissions to ICU is trivial as this information can directly be inferred from the medical records. Labeling the occurrence of hospital-acquired infections is slightly more involved since one must basically distinguish secondary infections occurring during hospital stay from infections existing before admission. For this purpose, medical experts guided us to label complications in terms of the International Classification of Diseases, Ninth Revision, Clinical Modification (ICD-9-CM) codes [6] that are used in medical records, inspired from the work of Roosan et al. [7]. We implemented complication labeling as a one-pass algorithm that labels each admission with the complication(s) that occurred a posteori (if any). This served to establish a ground truth, which we use for training models.

\subsubsection{Participants and Occurrence of Complications}

Figure 1 illustrates the distribution of eligible admissions by age and gender. The gap in the number of admissions between genders for people aged between 15 and 40 years is due to pregnancies. The fact that females tend to live longer explains the gap in the number of admissions of older people.

${ }^{4} 171892$ people were admitted to ICU on the first day: they have been excluded from the train and test population for the ICU label. 


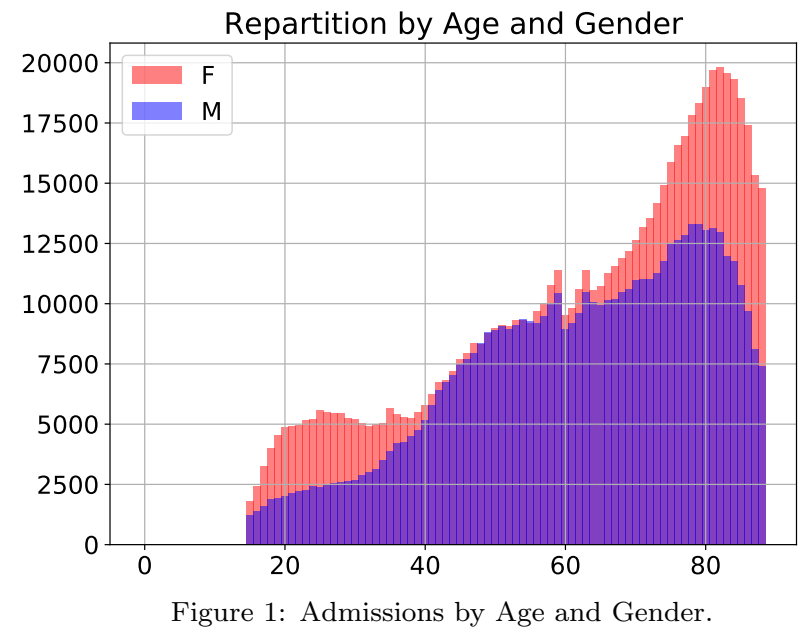

Among the overall population, there were 39988 cases of hospital death (3.14\%), 34076 cases of pressure ulcers complications (2.68\%), 45542 cases of ICU admission on or after the second day (3.58\%), and 32198 cases of hospital-acquired infections $(2.53 \%)$. On average, the probability that a patient experiences during his hospital stay at least one of the considered complications is $10.43 \%$.

Figure 2 shows the percentage of occurrence of each complication for each of the 417 hospitals considered. The proportion of complications appears roughly

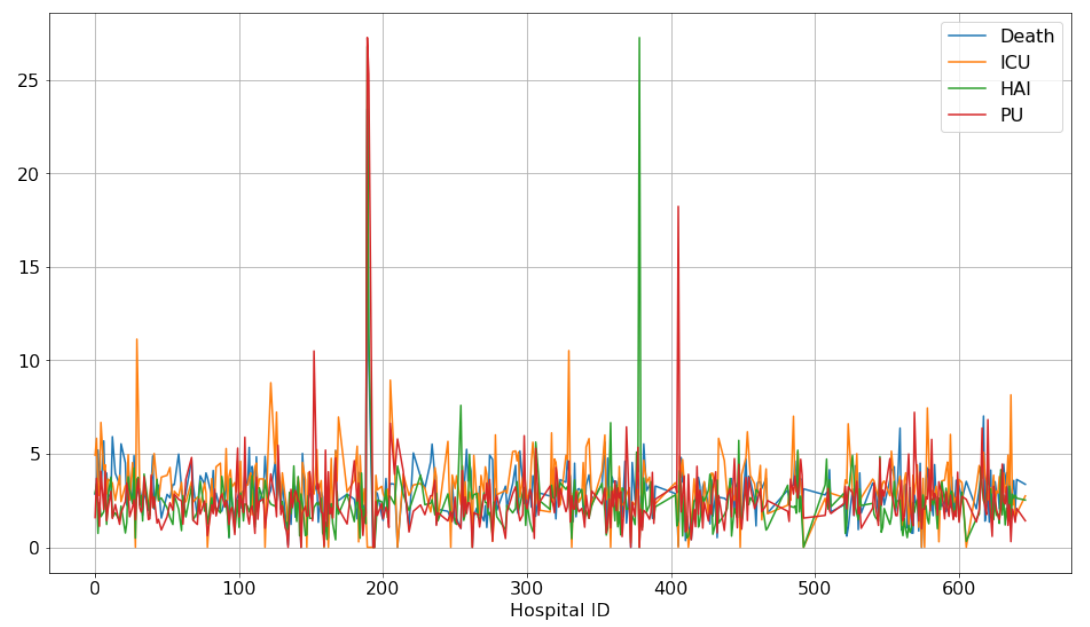

Figure 2: Risk (in \%) of Occurrence of Complications per Hospital.

similar between hospitals except for a few of them (shown by the peaks on Figure 2). The filters that we apply (see Section 2.1.1) retain a number of 
eligible admissions that varies between hospitals (leaving for instance 15859 relevant admissions for one hospital and 2 relevant admissions for another, with a mean number of 3568 relevant admissions per hospital). The peaks observed on Figure 2 actually correspond to hospitals having a significantly lower number of eligible admissions for our study. For instance, the most important peaks correspond to the following cases:

$\begin{array}{lllll}\text { Death } & \text { Hospital 189: } & 49 \text { out of } 209 \text { eligible admissions } & (23.4 \%) \\ \text { ICU } & \text { Hospital 29: } & 95 \text { out of } 853 \text { eligible admissions } & (11.1 \%) \\ \text { HAI } & \text { Hospital 378: } & 6 \text { out of } 22 \text { eligible admissions } & (27.3 \%) \\ \text { PU } & \text { Hospital 189: } & 57 \text { out of } 209 \text { eligible admissions } & (27.3 \%)\end{array}$

We consider that for these cases, the low numbers of eligible admissions explain the peaks observed. In the sequel, we abstract over the fact that some hospitals might be more prone to the development of complications than others. We concentrate on developing a predictive system intended to work with any hospital.

Figure 3 illustrates the distribution of drugs served. For each drug (on the $\mathrm{x}$-axis), it shows the (logarithmic) number of patients who were served the drug on their first day. This shows that, as one might expect, some drugs are served to a very large proportion of patients ${ }^{5}$ while other drugs are rarely served, and some almost never served. One goal of our predictive system is to automatically extract information from prescription data (including associations of drugs more or less frequently served) relevant for predicting the occurrence of complications. The purpose is to create information and risk signals usable by clinicians.

\subsection{Predictive System}

We now review the main principles and choices that we have made in designing the prediction system.

\subsubsection{Distributed Approach}

Distributing data and computations was instrumental for processing the aforementioned data ${ }^{6}$. We thus first review the distributed approach that made it possible to obtain our results. The structure of our prediction system is illustrated in Fig. 4. Initial data consist in a set of raw relational tables, that we store in a NFS distributed file system. This file system communicates with Spark SQL [8] that we use for data preprocessing, integration, and filtering. For optimizing the representation of features, we use a library for perfect hashing,

\footnotetext{
${ }^{5}$ For instance, the top three drugs that are the most served on the first day include respectively "0.9\% NACL 1000ML" (served 305293 times), "PANTOPRAZOLE, PROTONIX TAB 40MG" (served 153971 times), and "ACETAMIN, TYLENOL TAB 325MG" (served 122306 times).

${ }^{6}$ Initial attempts with the Pandas library on a single machine with 160 GB of RAM were non-conclusive. Only a fraction of the dataset was fitting in memory (after joining and filtering made using a distributed algorithm) and yet no transformation requiring copies (e.g. joins) was possible. We tried to compute joins by chunks and finally stopped the computation after 3 days (for an estimated time of at least 6 days).
} 


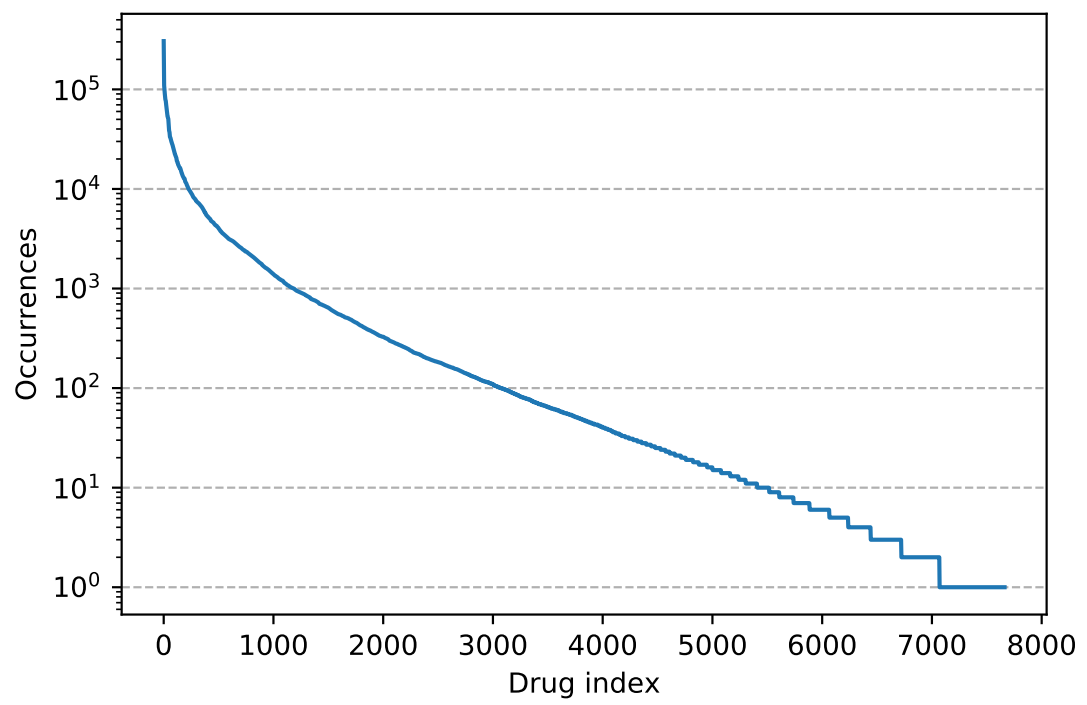

Figure 3: Distribution of drugs served (the y-axis indicates the number of patients to which each drug on the $\mathrm{x}$-axis was served).

that we modified and upgraded for use in our Spark and Python environments, based on the work of Czech et al. [9]. The feature engineering and classification components are hand coded in Spark [10] and SparkML [11]. We use distributed implementations of Logistic Regression (LR) [12], Linear Support Vector Machines (LSVM) [13], Decision Trees (DT) [14] and FP-Growth [15]. We also used t-SNE [16] and facilities provided by Pandas and Scikit-Learn libraries on smaller excertps of data that were extracted and preprocessed with Spark. We use Docker [17] to improve the runtime performance of the distributed architecture (mainly input/output) compared to a traditional approach with virtual machines. We automatically deploy custom Docker images on each machine of the cluster. The use of Docker also facilitates deployment on commodity and heterogenous machines. We use Jupyter Notebooks as a prototyping frontend.

Our cluster is composed of 5 machines each equipped with $2 \operatorname{Intel}(\mathrm{R}) \mathrm{Xeon}(\mathrm{R})$ from $1.90 \mathrm{GHz}$ to $2.6 \mathrm{Ghz}$, with 24 to 40 cores, and between $60 \mathrm{~GB}$ and $160 \mathrm{~GB}$ of RAM. The network is $1 \mathrm{~GB}$ ethernet.

\subsubsection{Feature Engineering}

The distributed architecture makes it possible to consider many features per patient. We have explored many combination of possible features, going from individual attributes such as the age and gender of a patient, to features describing in more or less details the drugs served on the first day for that patient. 


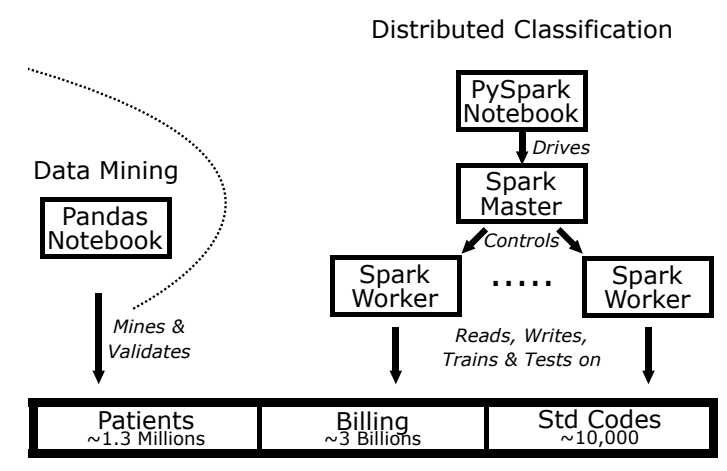

Figure 4: Architecture of the Prediction System.

For a given patient, we also consider a specific "score" often used in the clinical literature and readily available in hospital EHRs: the Medication Regimen Complexity Index (MRCI) [18]. MRCI is one of the most valid and reliable scale for assessing regimen complexity $[19]^{7}$. The MRCI score is meant to reflect the complexity of the patient's situation. The greater the MRCI, the more complex the patient's situation is. While the minimum MRCI score is 2 (e.g. one tablet taken once a day as needed), there is no maximum score. We retained MRCI as a marker of risk. We also include it in our study for the purpose of comparison with earlier works such as the one of Lepelley et al. [5].

We performed data mining on the dataset and used statistical techniques to search and select basic features among the population characteristics. For example, A 7.9\% overall correlation was found between patient's age and occurence of death during hospital stay. Figure 5 illustrates the distribution of MRCI levels among the considered population; and Figure 6 illustrates the percentage of people who expired at the hospital in terms of their MRCI level at admission. A $4.7 \%$ overall correlation was found between the MRCI value at admission and death at the hospital. In the sequel we investigate and report to which extent such correlations can actually be exploited for prediction purposes.

We tested different sets of features for building predictive models based on analyses of varying granularity. For the sake of clarity when reporting our

\footnotetext{
${ }^{7}$ Specifically, MRCI is a global score aggregating 65 sub-items for the purpose of indicating the complexity of a prescribed medication regimen. The MRCI has 3 sections giving information on the dosage form (section A), dosing frequency (section B) and additional instructions (section C) with 32, 23 and 10 items respectively. Each section reflects a different aspect of the complexity of prescription regimen. The total MRCI score is the sum of subscores for the 3 sections. MRCI is readily available in hospital EHRs but for our study, we had to recompute it from our prescription data. We computed it following the Appendix II of [19] which describes how to compute a score for each section. The only approximation is that our considered dataset lacks data required for computing the subscore for section $\mathrm{C}$, which we thus arbitrarily set to zero. In the sequel, the total MRCI score is thus the sum of sections A and $\mathrm{B}$.
} 


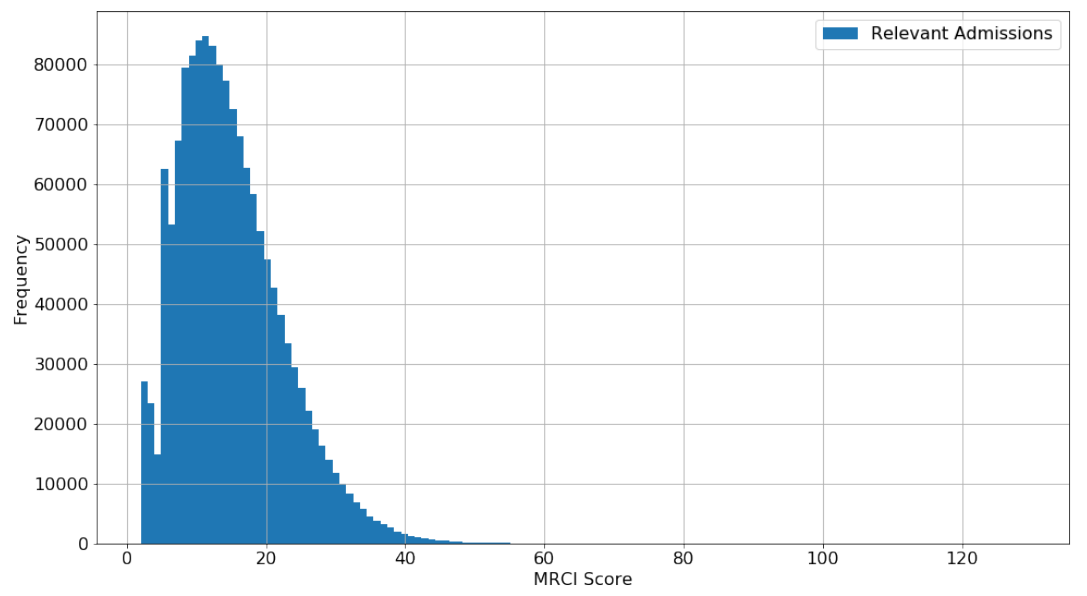

Figure 5: MRCI Levels in the Considered Population.

results, we define categories of features and use naming conventions.

For each admission, we concentrate on the following features, grouped by named categories:

- A list B of basic features including patient age, gender, and admission type (e.g. whether the patient is admitted from a doctor's office and requiring acute care for e.g. pneumonia or dehydration; or whether the patient in life-threatening condition such as accident victim).

- A score M that corresponds to MRCI at admission.

- A list $\mathrm{C}$ of clinical quantities associated to drugs prescribed on the first day. The length of $\mathrm{C}$ is the number of all drugs possibly prescribed during the first day in any hospital: there are more than 10 thousands such drugs. For example, Table 1 shows a sample vector including clinical quantities of drugs served on the first day. Since only a few drugs are served to each patient on the first day (compared to the length of $\mathrm{C}$ ), we adopt a sparse representation for features in memory. In addition, we also consider an alternative choice of feature representation where drugs are grouped into drug categories. A drug category regroups drug variants with e.g. different dosage. A category typically regroups 10 drug variants. Thus, as an alternative to the original list $\mathrm{C}$ we also consider a list $\mathrm{P}$ of integer values (with one integer per category of drugs) indicating the number of drugs served on the first day in the category. Specifically, with our dataset, C contains 10739 entries and P has 2046 entries.

Table 1 illustrates a sample feature vector for a 15 years-old patient, who was served 16 drugs on the day of admission.

We consider combinations of the aforementioned feature categories: for example $\mathrm{B}+\mathrm{M}$, simply denoted as BM, corresponds to the use of all basic features and 


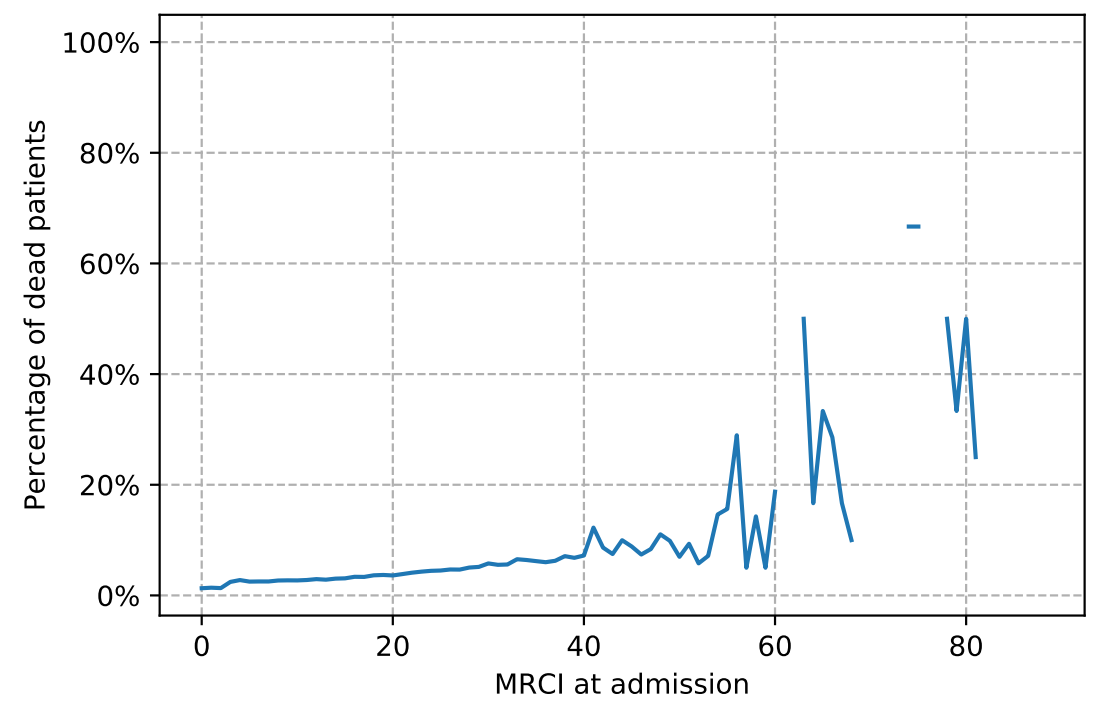

Figure 6: Risk of Death in terms of MRCI Level at Admission.

MRCI; whereas BMC also includes the features corresponding to clinical quantities of drugs served on the first day. In the sequel, we compare the predictive performances achieved with increasingly fine-grained models obtained with BM, BMP and BMC features for instance.

\subsubsection{Classifiers}

We conducted extensive tests with different classifiers including linear classifiers (LR, LSVM), decision trees and random forests. In the sequel, we mainly report on our experiments with the LR classifier to make predictions. The reason is that LR was the classifier that yielded the best predictive accuracy among several widely-used classifiers (See $\S 3.1$ for comparative metrics and Figures 7 and 8 for comparisons). Notice that LSVM also yields a similar predictive performance. For equivalent performances, we still favor LR because its raw output has a probabilistic interpretation. For our tests, we use the SparkML distributed implementation $[11,10]$ of the LR classifier [12].

Like several other standard machine learning methods, LR can be formulated as a convex optimization problem, i.e. the task of finding a minimizer of a convex function $f$ that depends on a variable vector $w$ which has $d$ entries. More formally this can be written as the optimization problem

$$
\min _{\boldsymbol{w} \in \mathbb{R}^{d}} f(\boldsymbol{w})
$$




\begin{tabular}{|c|c|c|c|}
\hline $\begin{array}{l}\text { Feature } \\
\text { Index }\end{array}$ & $\begin{array}{c}\text { Feature } \\
\text { Description }\end{array}$ & $\begin{array}{l}\text { Feature } \\
\text { Value }\end{array}$ & $\begin{array}{l}\text { Standard Charge } \\
\text { Master Code }\end{array}$ \\
\hline 0 & Age & 15 & \\
\hline 1 & Gender ( 1 for male, 0 for female) & 1 & \\
\hline 2 & MRCI & 24 & \\
\hline $802 \overline{4}$ & DEXTROSE/NACL SOLUTION $100 \overline{-} \bar{M}$ & 1.00 & $250 \overline{2} 5 \overline{8} 0 \overline{0} 0 \overline{97} \overline{0} 00 \overline{0}$ \\
\hline 7955 & NACL SOLUTION 100ML & 2.50 & 250258000220000 \\
\hline 7949 & NACL SOLUTION 1000ML & 1.00 & 250258000160000 \\
\hline 7084 & DOCUSATE NA CAP 100MG & 1.00 & 250257020020000 \\
\hline 6654 & ACETAMIN TAB 325MG (EA) & 2.00 & 250257000530000 \\
\hline 4869 & SOD BICARB INJ $8.4 \%$ 50MEQ 50ML & 1.00 & 250250058740000 \\
\hline 4332 & POT CHL VL 2OMEQ 10ML & 0.50 & 250250053100000 \\
\hline 3566 & MORPHINE TAB SR 3OMG & 0.50 & 250250044450000 \\
\hline 5871 & PEG-ES, MIRALAX PWDR 255GM & 0.07 & 250250100890000 \\
\hline 3483 & MISC TOPICAL & 1.00 & 250250043560000 \\
\hline 1563 & DIPHENHYD INJ 50MG & 1.00 & 250250019540000 \\
\hline 1292 & CYCLOBENZAPRINE TAB 10MG & 2.00 & 250250016100000 \\
\hline 2882 & LANSOPRAZOLE CAP 30MG & 1.00 & 250250036600000 \\
\hline 784 & CEFEPIME VL $1 \mathrm{GM}$ & 2.00 & 250250010280000 \\
\hline 3093 & LORATADINE TAB 10MG & 1.00 & 250250038770000 \\
\hline 134 & ALLOPURINOL TAB 100MG & 1.00 & 250250001580000 \\
\hline
\end{tabular}

Table 1: Feature vector for a sample patient who was served 16 drugs on the day of admission.

in which the objective function $f$ is of the form:

$$
f(\boldsymbol{w})=\lambda R(\boldsymbol{w})+\frac{1}{n} \sum_{i=1}^{n} L\left(\boldsymbol{w} ; \boldsymbol{x}_{\boldsymbol{i}}, y_{i}\right)
$$

where the vectors $\boldsymbol{x}_{\boldsymbol{i}} \in \mathbb{R}^{d}$ are the training data examples, for $1 \leq i \leq n$, and $y_{i} \in \mathbb{R}$ are their corresponding labels, which we want to predict; and the logistic loss function $L$ is of the form:

$$
L(\boldsymbol{w} ; \boldsymbol{x}, y)=\log \left(1+\exp \left(-y \boldsymbol{w}^{T} \boldsymbol{x}\right)\right)
$$

The purpose of the regularizer $R(\boldsymbol{w})$ is to encourage simple models and avoid overfitting. The fixed regularization parameter $\lambda$ defines the trade-off between the two goals of minimizing the loss (i.e., training error) and minimizing model complexity (i.e., to avoid overfitting). Our reported experiments were obtained with $L 2$ regularization, i.e. $R(\boldsymbol{w})=\frac{1}{2}\|\boldsymbol{w}\|_{2}^{2}$ and $\lambda=\frac{1}{2}$.

Given a new data point, denoted by $\boldsymbol{x}$, the LR model makes predictions by applying the logistic function:

$$
f(z)=\frac{1}{1+e^{-z}}
$$

where $z=\boldsymbol{w}^{T} \boldsymbol{x}$. We eventually use a threshold $t$ such that if $f\left(\boldsymbol{w}^{T} x\right)>t$, the outcome is predicted as positive, or negative otherwise. By default we choose $t=0.5$ unless specified otherwise. We make $t$ vary to compute ROC curves and 


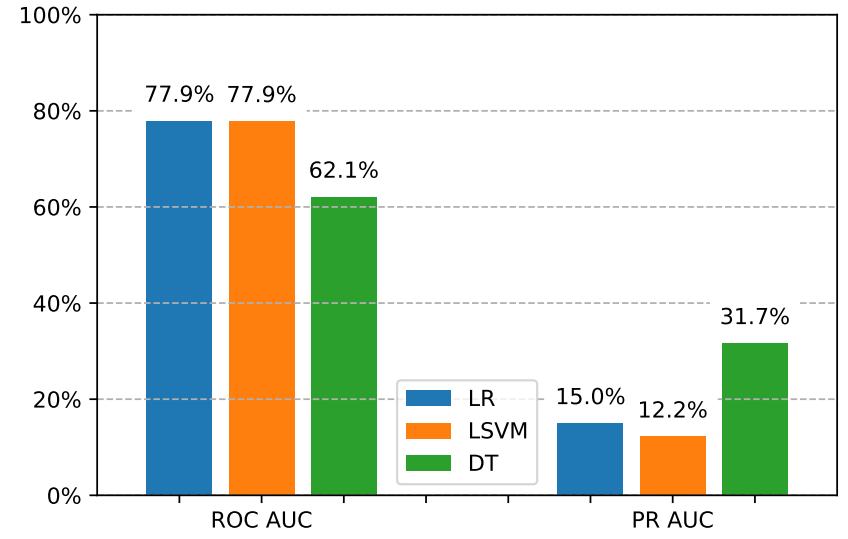

Figure 7: Performances of different classifiers for predicting hospital mortality on imbalanced (real) test sets.

report area under curves (See Section 3.1). Notice however that the raw output of the logistic regression model, $f(z)$, already has a probabilistic interpretation (i.e. the probability that $\mathrm{x}$ is positive).

\subsubsection{Cross-Validation, Class Imbalance, and Normalization}

We perform cross-validation: we separate training and testing subsets and we use only the training subset to fit the model and only the testing subset to evaluate the accuracy of the model. We pick the training and testing subsets randomly and in a disjoint manner. In practice we used at least 3-fold cross validations and up to 10 -fold cross-validations.

There are many more patients without complication than patients experiencing complications during their stays (hopefully). To deal with this class imbalance, we applied two different methods in order to rebalance classes before the random selection of the training subset: downsampling the set of patients with no complication, and learning with weighted coefficients (so that the impact of each instance is proportional to the overall class imbalance). We apply feature normalization for the linear models.

\section{Results}

We provide experimental evidence to back our claims on big prescription data. We bring novel insights concerning volume, variety, generality, velocity, scalability, and explainability in the construction of predictive models for complications. For assessing the quality of predictive models, we rely on a set of performance metrics that we first introduce. 


\subsection{Performance Metrics}

For a given complication, our system outputs a boolean prediction (either positive or negative) for each admission. To evaluate prediction results, we use recall, precision and other standard metrics computed from confusion matrices $[20,21]$. In particular, we use the area under the ROC curve (ROC AUC) evaluated on the test data, which is the standard scientific accuracy indicator [22]. The higher AUC indicates the better prediction performance. Intuitively, when using normalized units, AUC is equal to the probability that a classifier will rank a randomly chosen positive instance higher than a randomly chosen negative one [20]. We also use the area under the precision-recall curve (PRAUC) as an additional insight (though for any dataset, the ROC curve and PR curve for a given algorithm contain the same points [23]). We use precision, recall, ROC AUC, and PR-AUC to evaluate the overall predictive performance in terms of a large variety of features and a large volume of training data.

\subsection{Variety}

We investigate the impact of considering more or less fine-grained features when predicting complications. In other terms, we examine whether considering more features (Variety) per instance yields a better predictive accuracy.

We consider the list of basic features (B) for each patient, the MRCI score (M), clinical quantities (C), and combinations of them. Fig. 8 presents ROC curves and AUC results for mortality prediction. Fig. 9 presents ROC curves and AUC results for the prediction of pressure ulcers. ROC AUC is greater than $80 \%$ with BMC features which is significantly greater than with BM features $(63 \%)$.

The finer-grained features we consider the better predictive performance we obtain; the best predictive performance being obtained with the combination of all features (BMC). In particular, we observe that the detailed clinical quantities yield a significant increase in predictive performance compared to basic features and MRCI (Fig. 8). We obtain similar gains when predicting other complications. These results confirm that Variety (the number and granularity of features) can significantly improve the predictive modeling accuracy.

\subsection{Volume}

We study the impact of data volume on the construction of models.

First, we study the impact of increasing the sizes of both the train and test subsets. For this purpose, we choose a constant size ratio between the train subset and the test subset. We set this ratio to $2: 1$, meaning that we construct models from a train dataset whose size is the double of the size of the test subset. We make the sizes of both the train and test subsets vary while keeping this constant 2:1 ratio between their respective sizes. The results in terms of ROC AUC are presented in Figure 10a. Results in terms of recall are shown in Figure 10b.

Second, we study the impact of a dramatic increase in the number of training instances on predictive modeling accuracy. For this purpose, we randomly pick 


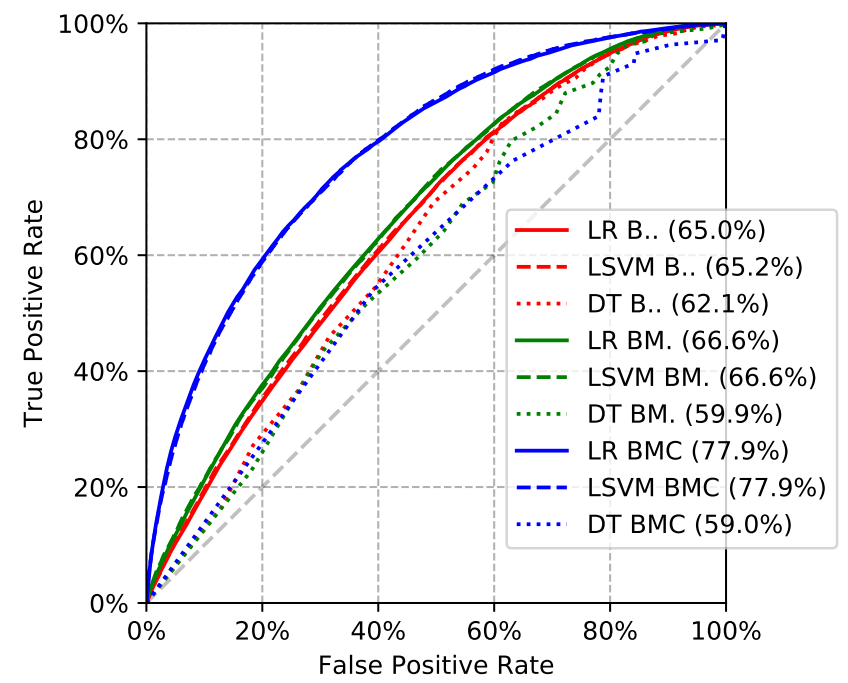

Figure 8: Impact of Finer-Grained Features (Variety) on ROC AUC for Predicting Mortality.

a test dataset that we keep constant while we repeatedly construct models with training datasets of varying sizes. We recall that in all our tests there is no overlap between the train and test datasets which are always chosen randomly in a disjoint manner.

Figures 11a, 11b and 12 present results in terms of ROC AUC, Recall and PR AUC (respectively) for the same test dataset with train datasets of increasing sizes. All models are evaluated on the same randomly chosen test subset of around 3010 instances, while we increase the train dataset size, as reported on the x-axes of the graphs of Figures 11a, 11b and 12 indicating the size of the training set $(100 \%$ corresponding to a train dataset of around 1267000 instances, $75 \%$ corresponding to around 950000 instances in the train dataset and $10 \%$ corresponding to around 127000 instances). The test subsets are randomly chosen and extracted from the remaining part of the full initial dataset (after removal of the test dataset).

We observe that increased volume tends to improve predictive performance. The availability of prescription data in very large volumes is beneficial for predicting complications.

\subsection{Generality in Predicting Complications}

We further investigate our initial postulate that drug prescription data on the day of admission contain rich information about the patients situation and perspectives of evolution. We study to which extent this information is general i.e. whether it can effectively be extracted for predicting different complications. For this purpose, we make our system builds (learns) a specific model for each 


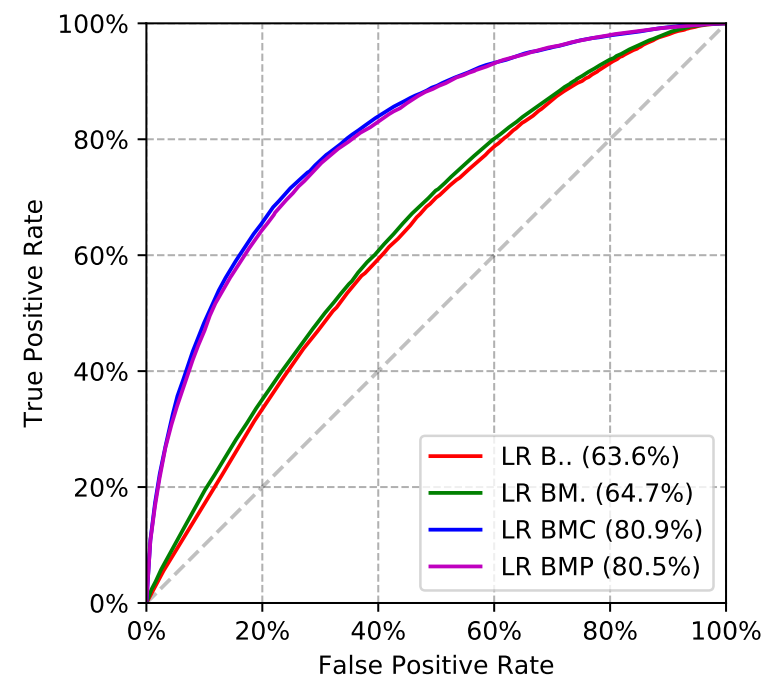

Figure 9: Impact of Finer-Grained Features (Variety) on ROC AUC for Predicting PU.

complication and we assess the quality of models. We now examine and evaluate the predictions for the different complications that we consider. We performed extensive tests using cross-validation methodology (see $§ 2.2 .4$ ), and we report on accuracy obtained from randomly chosen training and (disjoint) testing sets.

Figure 13a shows a ROC curve obtained when predicting hospital death. We obtain a ROC AUC greater than $76 \%$.

ROC curves and AUC obtained when predicting occurrence of pressure ulcers are shown on Figure 13b. Figure 13c shows the results for ICU admissions, and Figure 13d the results for hospital-acquired infections.

Overall, the system exhibits best accuracy for predicting the occurrence of hospital-acquired infections, pressure ulcers, and hospital deaths. Table 2 further illustrates detailed metrics on randomly selected datasets, with a threshold $t=0.5$.

\subsection{Velocity}

We report on how fast the models can be generated with respect to the considered dataset size.

Three subtasks are particularly computationally-intensive: (i) the data preprocessing including prefiltering, joining and feature extraction from data (as explained in $\S 2.1 .1$ and 2.2.2), (ii) the construction (learning) of models (see $\S 2.2 .3$ ), and (iii) the evaluation of the model over a test dataset. Notice that since we perform cross-validation, the two latter steps are often grouped and performed repeatedly. Afterwards, once a model has been computed, its execution (for computing predictions) is very efficient. A cluster of machines is 


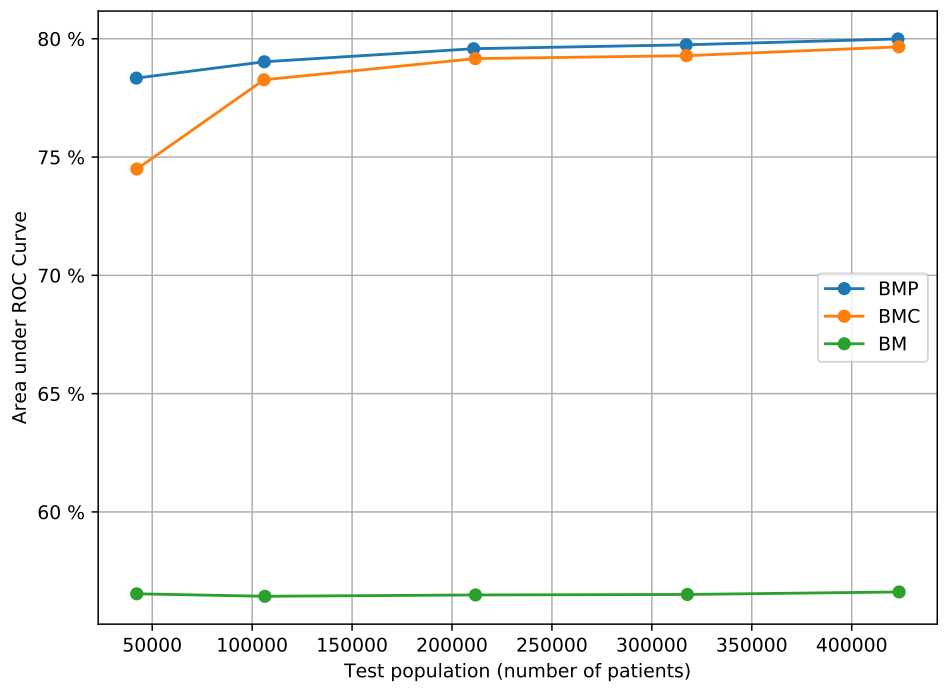

(a) Increasing volume leads to better ROC AUC.

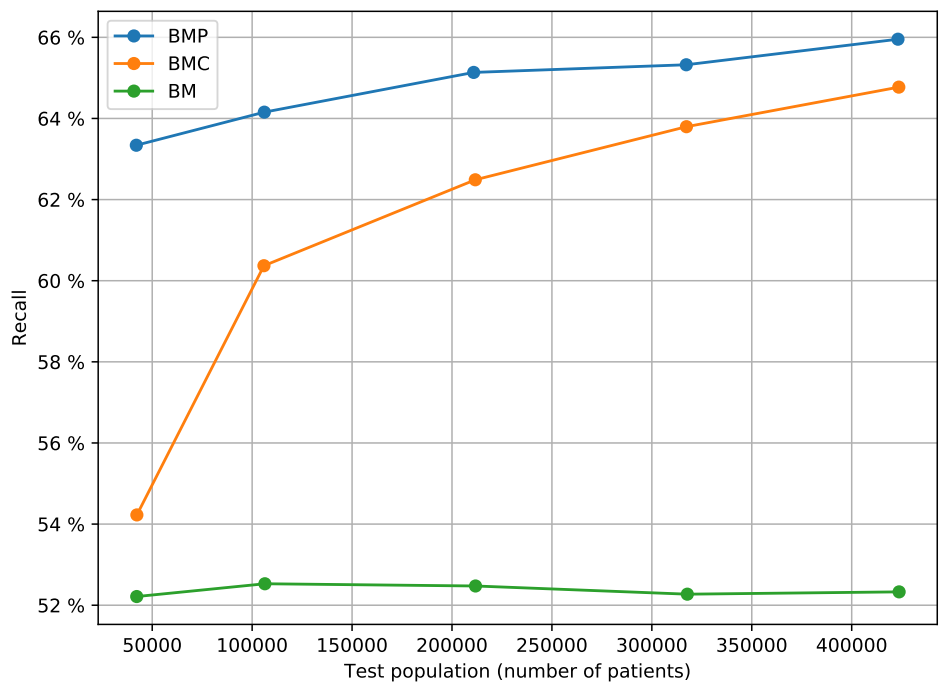

(b) Increasing volume leads to better recall.

Figure 10: Impact of volume on predictive performance with train and test sets of varying size (but with constant 2:1 ratio). Prediction of HAI. 


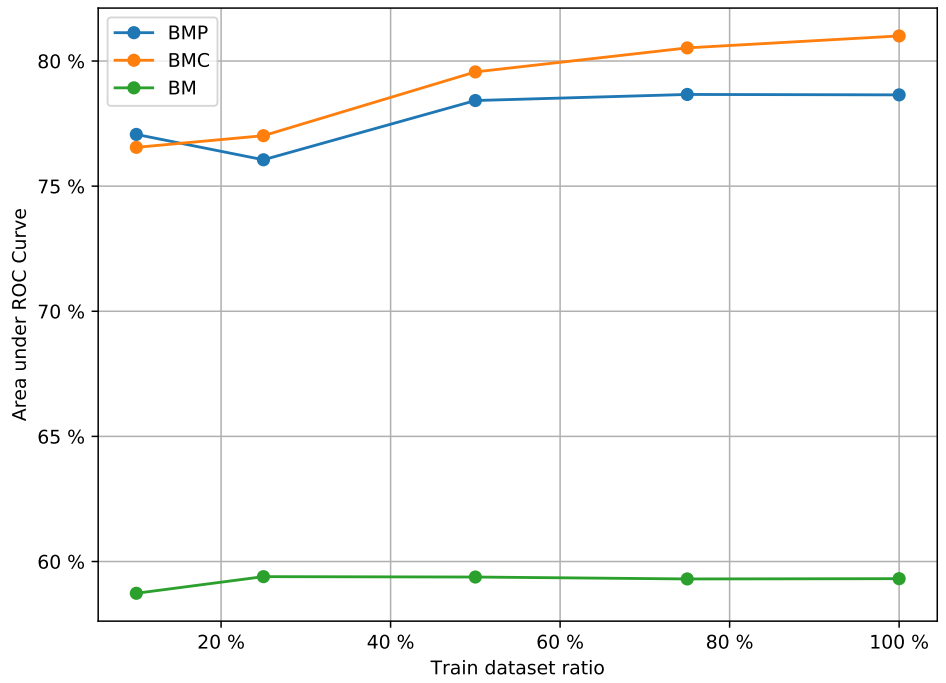

(a) Increasing volume tends to yield better ROC AUC.

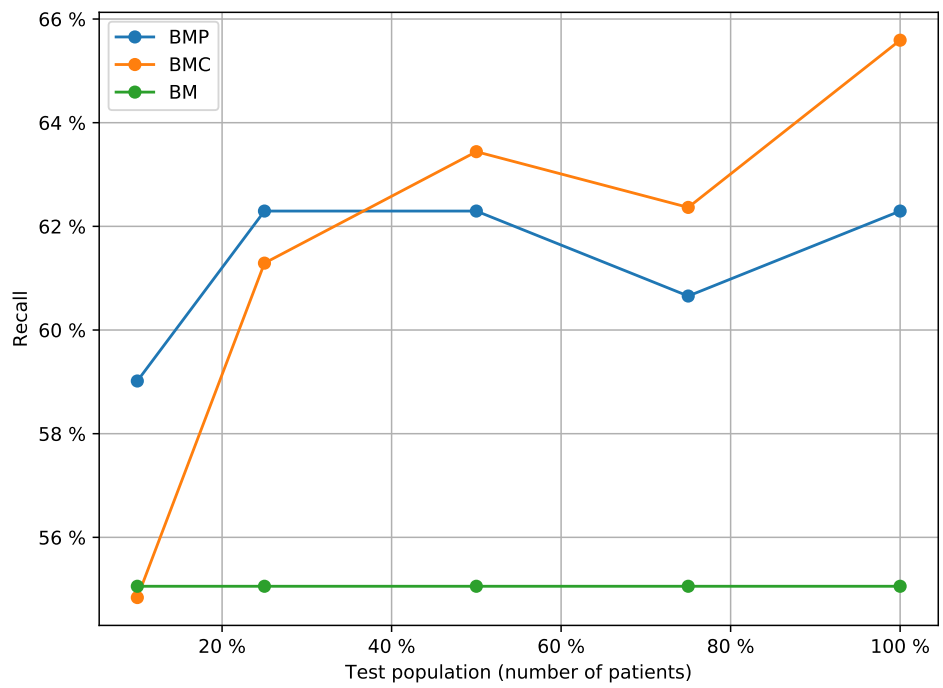

(b) Increasing volume tends to yield better recall.

Figure 11: Impact of volume on predictive performance with train sets of varying sizes, and same test set. Prediction of HAI. 


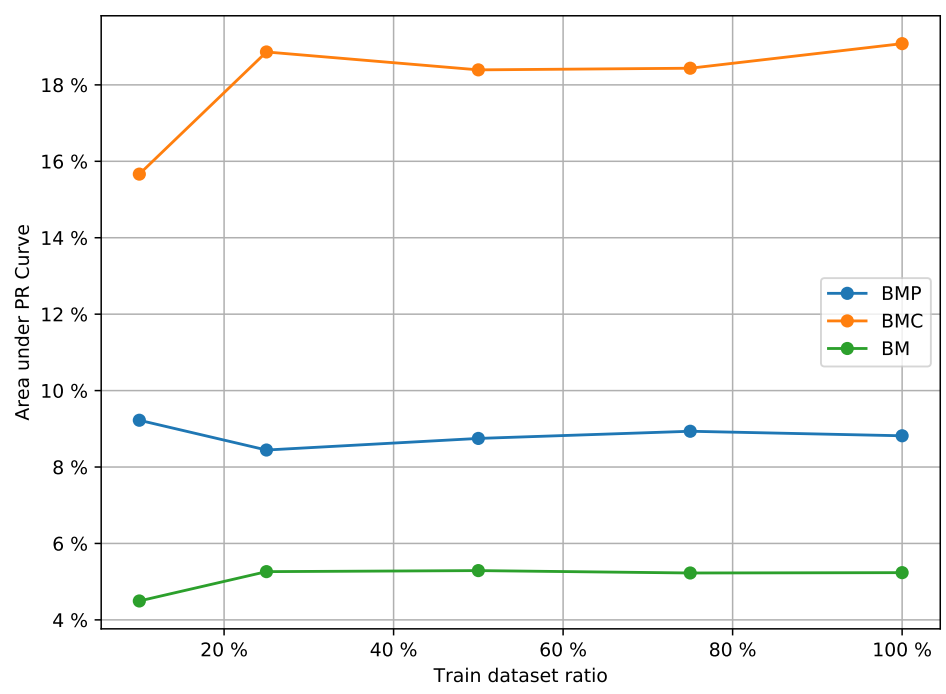

Figure 12: PR AUC (train subsets of varying sizes and same test subset), when predicting hospital-acquired infections. Notice that classes are heavily imbalanced: hospital-acquired infections occur for 3\% of all admissions. Metrics are reported for imbalanced (real) test sets.

then no longer needed: the model can be saved and transmitted (e.g. in PMML standard format) to be executed on a single commodity machine.

A BM model is computed in approximately 3.5 seconds from a training dataset of $800 \mathrm{~K}$ instances, validated in 8 seconds on a test dataset of $400 \mathrm{~K}$ instances, and executed in a negligible amount of time (a few ms) on a single instance (to obtain a prediction).

A more sophisticated BMP model (with more then 3000 features) is computed in 20 seconds from a training dataset of $800 \mathrm{~K}$ instances, validated in 14 seconds on a test dataset of $400 \mathrm{~K}$, and executed in a negligible amount of time (a few $\mathrm{ms}$ ) on a single instance.

A BMC model (with more than 10000 features) is computed in 108 seconds and validated in 29 seconds on datasets of similar sizes, and also executed in a negligible amount of time on a single instance.

Figure 14 illustrates the elapsed computation times in terms of the dataset size, for the different kind of models (from the simplest BM ones to the more complex BMP and BMC ones.

For the aforementioned computations to be possible, an additional one-time preprocessing stage is necessary to load and filter data (15s for loading and filtering patient data, and 4 minutes for loading, filtering and joining with billing data stored in CSV format). This preprocessing stage is done only once; afterwards and we restart from intermediate data that we store in the Parquet 


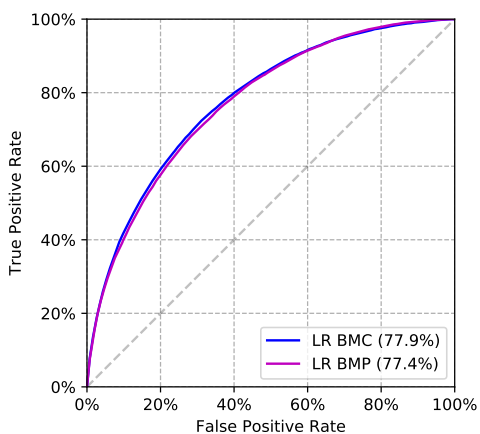

(a) Predicting Death (AUC $\geq 77 \%$ ).

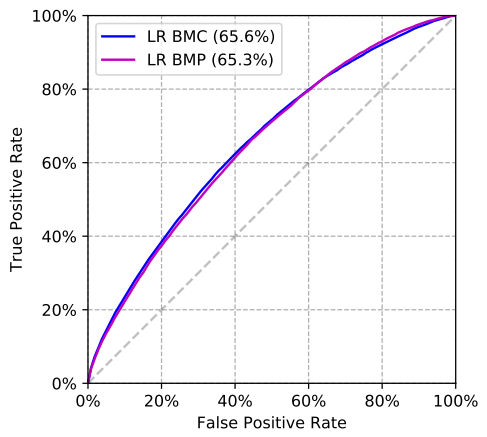

(c) ICU Admissions (AUC $\geq 65 \%$ ).

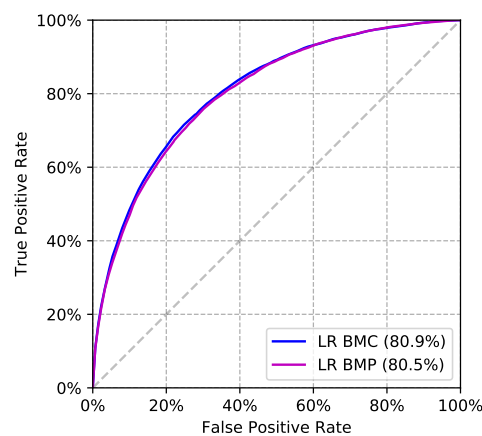

(b) Pressure Ulcers (AUC $\geq 80 \%$ ).

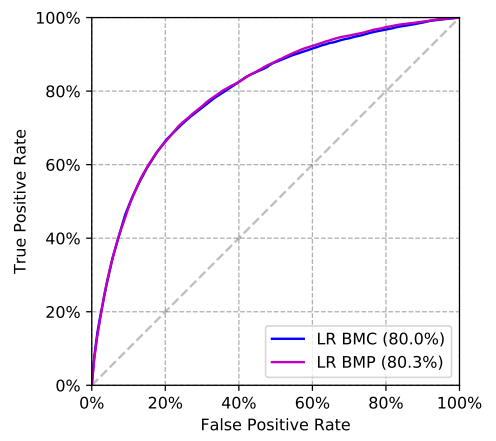

(d) HAI $($ AUC $\geq 80 \%)$.

Figure 13: Predicting Hospital Death, Pressure Ulcers, ICU Admissions and Hospital-Acquired Infections with BMC and BMP Features (randomly picked datasets). 


\begin{tabular}{rrrrrl}
\hline Metric & Death & ICU & PU & HAI & Metric Definition \\
\hline True Positive Rate & $65.1 \%$ & $58.1 \%$ & $68.4 \%$ & $65.7 \%$ & TP/P \\
True Negative Rate & $75.4 \%$ & $64.1 \%$ & $78.0 \%$ & $80.5 \%$ & TN/N \\
False Positive Rate & $24.6 \%$ & $35.9 \%$ & $22.0 \%$ & $19.5 \%$ & $\mathrm{FP} / \mathrm{N}$ \\
False Negative Rate & $34.9 \%$ & $41.9 \%$ & $31.6 \%$ & $34.3 \%$ & $\mathrm{FN} / \mathrm{P}$ \\
Negative Predictive Value & $98.5 \%$ & $97.3 \%$ & $98.9 \%$ & $98.9 \%$ & $\mathrm{TN} /(\mathrm{TN}+\mathrm{FN})$ \\
Positive Predictive Value & $7.9 \%$ & $6.5 \%$ & $7.9 \%$ & $8.1 \%$ & $\mathrm{TP} /(\mathrm{TP}+\mathrm{FP})$ \\
False Discovery Rate & $92.1 \%$ & $93.5 \%$ & $92.1 \%$ & $91.9 \%$ & $\mathrm{FP} /(\mathrm{TP}+\mathrm{FP})$ \\
Accuracy & $75.1 \%$ & $63.9 \%$ & $77.8 \%$ & $80.2 \%$ & $(\mathrm{TP}+\mathrm{TN}) /(\mathrm{P}+\mathrm{N})$ \\
Error & $24.9 \%$ & $36.1 \%$ & $22.2 \%$ & $19.8 \%$ & $(\mathrm{FP}+\mathrm{FN}) /(\mathrm{P}+\mathrm{N})$
\end{tabular}

Table 2: Detailed LR prediction metrics ${ }^{a}$ on random train and test subsets, with notations adopted from Fawcett [20]: TP is the number of true positives, FP: false positives, TN: true negatives, $\mathrm{FN}$ : false negatives, $\mathrm{P}=\mathrm{TP}+\mathrm{FN}$ and $\mathrm{N}=\mathrm{FP}+\mathrm{TN}$.

Notice that classes are heavily imbalanced since complications typically occur for around $3 \%$ of all admissions. The presented metrics are computed on imbalanced (real) test subsets.

${ }^{a}$ True Positive Rate is also known as Hit Rate, Recall, and Sensitivity; True Negative Rate is also known as Specificity; False Positive Rate as Fall-out; False Negative Rate as Miss Rate; and Positive Predictive Value as Precision.

format (which can be loaded in less than 10s). All these performance figures are obtained with the cluster of 5 machines set up to use 48GB of RAM per executor and 128 cores.

\subsection{Scalability}

We report on the extent to which model construction benefits from the availability of greater computational resources in the cluster of machines.

We make the computational resources of our cluster vary and study running times depending on the amount of memory available per executor and the total number of cores in the cluster. Figure 15a shows the elapsed times spent for a 3 -fold cross validation process of a BM model depending on the cluster resources used. Each single time shown on Figure 15a thus corresponds to the total running time spent for 3 iterations of the construction and evaluation of a BM model on three randomly chosen datasets. Times are reported depending on two varying cluster resources: the amount of RAM per executor and the total number of cores available in the cluster. Figure 15a shows elapsed times when constructing BM models and Figure 15a shows elapsed times when constructing the more complex BMC models. Figure 16 illustrates the time variability of a single step of the cross-validation process (including time spent in splitting, normalizing features, training the model on one dataset, and evaluating the model on one dataset).

Figures all illustrate similar variations of computation times. Computation times decrease with the number of cores. We observe a particularly sharp decrease in computation time when increasing the number of cores from 2 to 24 . This overall behavior is similar independently of the amount of RAM available in each executor. We also observe that the computations tend to be faster when 


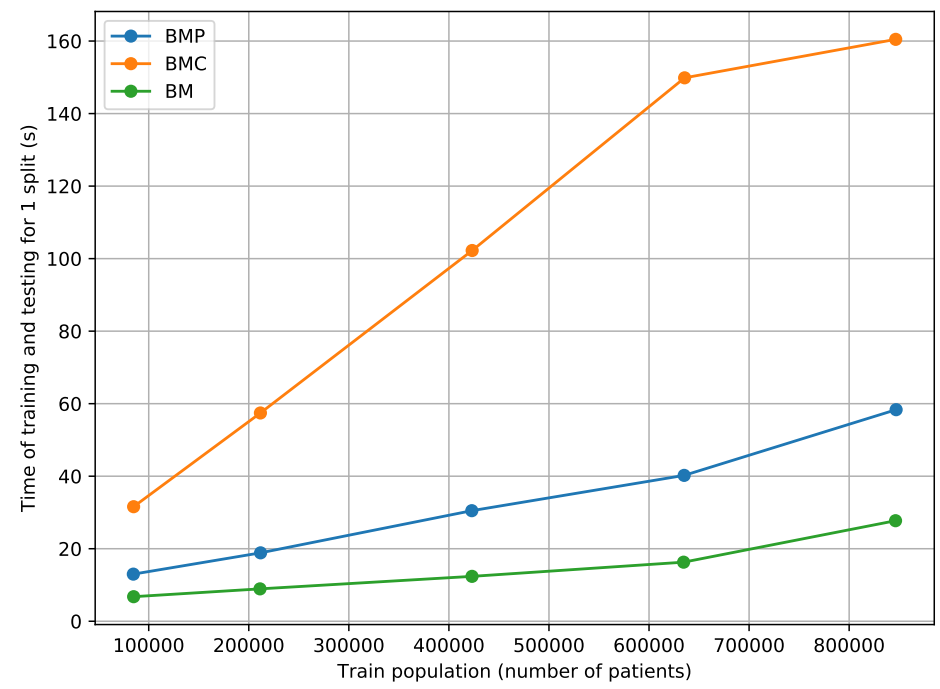

Figure 14: Times spent for constructing and testing models depending on dataset sizes.

more memory available in each executor. Overall, results show that the construction of simple and complex models greatly benefits from the distribution and the availability of more computational resources (mainly the number of cores) in the cluster.

\subsection{Explainability}

We pay particular attention to the explanability of the models that we generate. In other terms, we do not only focus on numerical predictive modeling accuracy but also concentrate on generating models that offer opportunities for further clinical interpretation and understanding. Model explainability helps in building clinical knowledge and may guide the search of further models.

We thus further study the weights of the LR models that we generate. The weights of an LR model represent a summarization of the respective importance of features over the training dataset. As such, it is thus interesting to study how stable is this summarization with respect to different training datasets.

We constructed BMP models to predict hospital-acquired infections for 108 randomly selected train sets, and analysed the weights of all models. Specifically, we concentrate on the top 10 most important positive weights. In other terms, we retain the set $S$ of all features $f$ that such that $f$ occurs in the top 10 most important positive weights in at least one of the 108 models. Only 12 drugs occur in $S$. These drugs are shown on Table $3 \mathrm{a}$ which presents the corresponding features sorted by their number of occurrences, and their identifier (not by weight which would require aggregation of some sort). We performed a similar analysis for the top 10 most important negative weights. Only 14 drugs form the most important negative weights, as shown in Table $3 \mathrm{~b}$. 


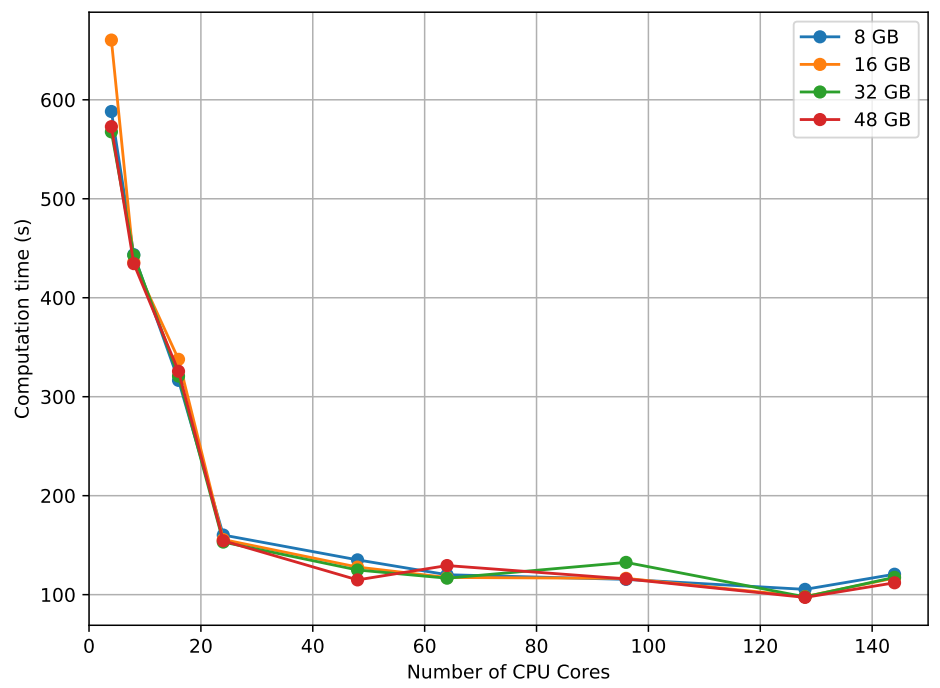

(a) Construction of BM models.

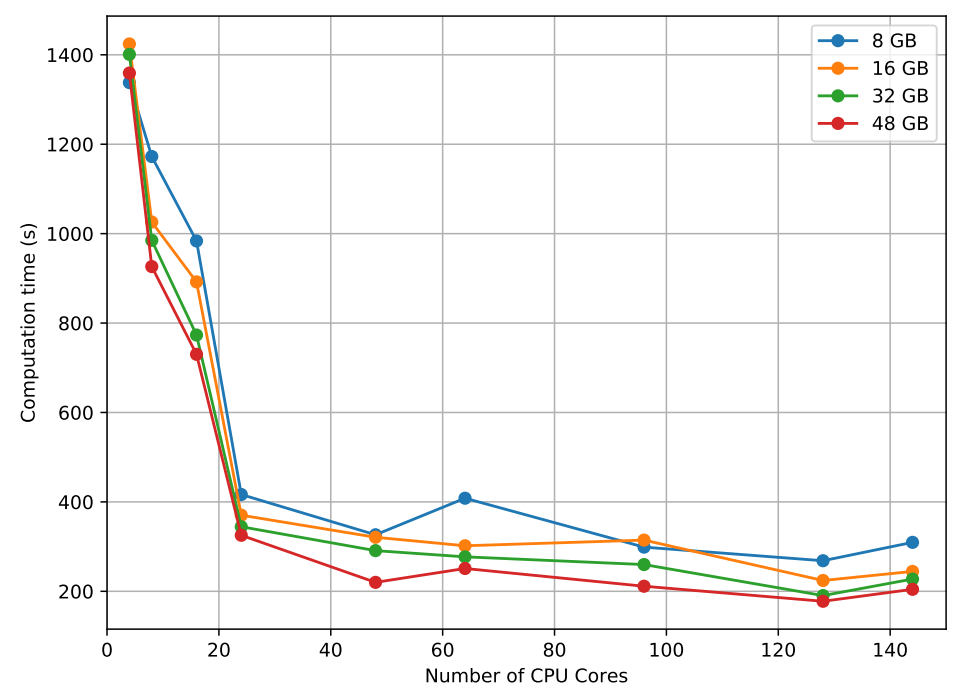

(b) Construction of BMP models.

Figure 15: Times spent for the construction of models depending on cluster resources. 


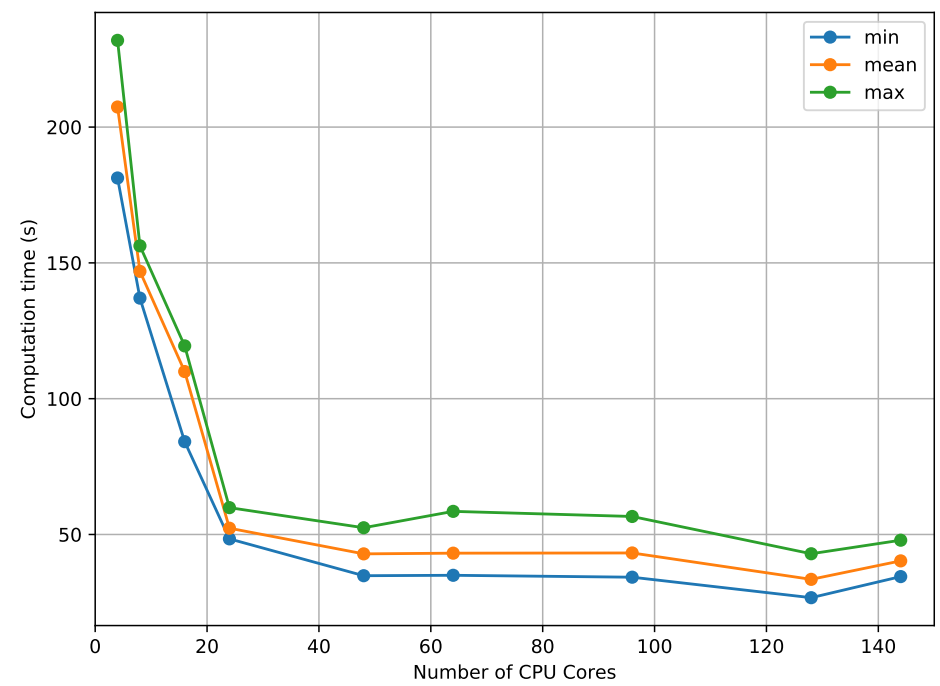

Figure 16: Times spent for a single step of the cross-validation process.

We also constructed BMC models for 102 randomly selected train sets. We analysed the weights of all models. This time, since these models use many more features, we pay attention to the topmost 20 positive and negative weights. Results are shown in Table 4 and 5, respectively.

We make the following observations:

- only the features indicated in the aforementioned tables form the most important weights of all models (there is no feature left with respect to the definition above);

- the majority of features retained this way appear in the most important weights of all models;

- the same obervations hold for the BMP and for the BMC models.

This illustrates the stability of weights and the robustness of the generated models with respect to the randomly selected train sets, opening the way for further clinical interpretation (beyond the scope of this article).

\section{Related Works}

A general overview of recent developments in big data in the context of biomedical and health informatics can be found in Pérez et al. [24]. With the broad adoption of EHRs systems, the development of techniques for improving the quality of clinical care has received considerable interest recently, especially from the AI community $[25,26,27,28,29]$. 


\begin{tabular}{|l|r|}
\hline Most Important Positive Features & Count \\
\hline 25025001018_CEFAZOLIN & 108 \\
25025002863_GENTAMICIN & 108 \\
25025004905_OXYCOD/ASA & 108 \\
25025005262_PIPERACILLIN/TAZO & 108 \\
25025006583_VANCOMYCIN & 108 \\
25025700053_ACETAMINOPHEN & 108 \\
25025800083_DEXTROSE SOLUTION & 108 \\
25025001030_CEFEPIME & 90 \\
25025800016_NACL & 83 \\
25025003050_HEPARIN NA FLUSH & 72 \\
25025000337_AMPICILLIN/SULBAC & 43 \\
25025003226_HYDROMORPHONE & 36 \\
\hline
\end{tabular}

(a) Positive Features

\begin{tabular}{|l|r|}
\hline Most Important Negative Features & Count \\
\hline 25025000520_AZITHROMYCIN & 108 \\
25025002815_FUROSEMIDE & 108 \\
25025003499_IPRATROPIUM & 108 \\
25025004224_METHYLPRED NA & 108 \\
25025004756_NITROGLYCERIN & 108 \\
25025004929_OXYTOCIN & 108 \\
25025700431_ASPIRIN & 108 \\
25025706196_VIT B1(THIAMINE) & 108 \\
25025000135_ALBUTEROL & 106 \\
25025001926_DINOPROSTONE & 60 \\
25025001966_DIPHTHERIA/TETANUS & 33 \\
25025704695_NICOTINE & 14 \\
25025003675_LEVALBUTEROL & 2 \\
25025003884_LORAZEPAM & 1 \\
\hline
\end{tabular}

(b) Negative Features

Table 3: The most important features in BMP models and the number of times they occur in the top 10 list of the most important weights, out of 108 randomly selected train sets.

\begin{tabular}{|l|r|}
\hline Most Important Positive Features & Count \\
\hline 250250065940000_VANCOMYCIN VL 500MG & 102 \\
250250065800000_VANCOMYCIN VL 500MG & 102 \\
250258001390000_DEXTROSE SOLUTION 100ML & 102 \\
250258000220000_NACL SOLUTION 100ML & 102 \\
250257000530000_ACETAMIN TAB 325MG (EA) & 102 \\
250250065950000_VANCOMYCIN VL 500MG & 102 \\
250250008220000_CA ACET TAB 667MG & 102 \\
250250065840000_VANCOMYCIN VL 500MG & 102 \\
mrCi & 102 \\
250250052870000_PIPERACILLIN/TAZO VL 2/0.25GM & 102 \\
250250052630000_PIPERACILLIN/TAZO VL 2/0.25GM & 102 \\
250250049010000_OXYCOD/ASA TAB 4.5MG/325MG & 102 \\
250250028630000_GENTAMICIN VL 40MG/ML 2ML & 102 \\
250250010180000_CEFAZOLIN VL 1GM & 102 \\
250250010130000_CEFAZOLIN VL 1GM & 102 \\
adm_tyPe_2 & 101 \\
250250003370000_AMP/SULBAC VL 3GM & 94 \\
250250100930000_SEVELAMER, RENAGEL CAP 403MG & 87 \\
250258000190000_NACL SOLUTION 10OML & 78 \\
250258001450000_DEXTROSE SOLUTION 10OML & 69 \\
250258000270000_NACL SOLUTION 10OML & 50 \\
250250010300000_CEFEPIME VL 1GM & 20 \\
250257000510000_ACETAMIN SUPP 325MG & 11 \\
\hline
\end{tabular}

Table 4: The most important positive features in BMC models and the number of times they occur in the top 10 list of the most important positive weights, out of 102 randomly selected train sets. 


\begin{tabular}{|l|r|}
\hline Most Important Negative Features & Count \\
\hline 250250001200000_ALBUTEROL INH SOL 0.5\% 1ML (5MG) & 102 \\
250250028190000_FUROSEMIDE VL 40MG 4ML & 102 \\
250257004340000_ASPIRIN TAB 325MG (EA) & 102 \\
250250105290000_OXYTOCIN VL 10U/ML 1ML & 102 \\
250250049270000_OXYTOCIN VL 10U/ML 1ML & 102 \\
250250047520000_NITROGLYCERIN OINT 2\% 1GM & 102 \\
250250042270000_METHYLPRED NA VL 125MG & 102 \\
250250035010000_IPRATROPIUM INH SOL 0.02\% 2.5ML & 102 \\
250250042240000_METHYLPRED NA VL 125MG & 102 \\
250250005200000_AZITHROMYCIN TAB 25OMG & 102 \\
250250005110000_AZITHROMYCIN VL 500MG & 102 \\
250250019660000_DIPHTHERIA/TETANUS ADLT INJ 0.5ML & 102 \\
250250019260000_DINOPROSTONE VAG SUPP 10MG & 102 \\
250257004310000_ASPIRIN TAB 325MG (EA) & 101 \\
250257046890000_NICOTINE PATCH 21MG/DAY & 99 \\
250250036760000_LEVALBUTEROL, XOPENEX INH SOL 0.63MG/3ML 3ML & 93 \\
250250029250000_GUAIFEN SYRP 10OMG/5ML 5ML & 64 \\
250250100600000_IPRATROPIUM/ALBUTEROL INH SOL 3ML & 64 \\
250250049290000_OXYTOCIN VL 10U/ML 1ML & 63 \\
250250052970000_PANTOPRAZOLE, PROTONIX I.V. VL 4OMG & 57 \\
250257061960000_VIT B1(THIAMINE) TAB 10OMG & 53 \\
age & 50 \\
250250001210000_ALBUTEROL INH SOL 0.5\% 1ML (5MG) & 34 \\
250250043570000_MISOPROSTOL TAB 200MCG & 14 \\
250250028170000_FUROSEMIDE VL 40MG 4ML & 13 \\
250250028180000_FUROSEMIDE VL 40MG 4ML & 5 \\
250250021370000_ENOXAPARIN INJ 30MG 0.3ML & 4 \\
\hline
\end{tabular}

Table 5: The most important negative features in BMC models and the number of times they occur in the top 10 list of the most important negative weights, out of 102 randomly selected train sets.

The work of Luo et al. [28] adresses ICU mortality risk prediction with unsupervised feature learning techniques from timeseries of physiologic measurements (whereas we consider supervised techniques on prescribed drug data at admission). Lee et al. [25] introduce a method for the purpose of extracting phenotype information from EHRs and for providing analyses on phenotypes. Kuang et al. [26] propose a baseline regularization model for the task of finding new indications for existing drugs leveraging heterogeneous drug-related data based on EHRs. Li et al. [27] explore joint models for extracting mentions of drugs and their side effects, such as diseases that they cause. Zhang et al. [29] explore survival prediction with a focus on intermittently varying data.

Closely related works in terms of research objectives are the independent works performed simultaneously found in Avati et al. [30], Rajkomar et al. [31]. Authors develop a model for predicting all-cause mortality of patients from EHR data. The research objectives pursued are similar, and the methodology and the data used exhibit some similarities but with several important differences. First, [30] and [31] require "longitudinal" data in the sense that for making a prediction, their systems require historical data for the patient: it relies on historical observation windows and several observation slices: [30] uses 4 observation slices and [31] uses data from 24 hours before hospital admission (and also after admission). Both systems require the knowledge of the patient situation at several moments in time. In sharp contrast, our predictive system only 
requires the list of drugs prescribed on the very first day of arrival at the hospital for making a prediction. Our prediction system is thus much more flexible and general, since it can be used with patients admitted for the first time with no known history. Second, [30] and [31] use deep neural network models whereas the current article presents results obtained with more classical models that: (i) perform faster on CPU machines, and (ii) are more intelligible: they offer more insights into the factors that influence the predictions, as emphasized in [32]. For instance, the weights computed by our models are stable and intelligible, allowing further interpretation (and fix if needed) by clinicians. Last but not least, to be able to operate without the need for historical data of patients, our models were trained with much more "transversal" data. [30] studies a population of 221284 patients (216 221 hospitalizations were studied in [31]), whereas our models are built from the automated analysis of more than 1.2 million of patients.

In fact, our work fundamentally differs from all these previous works by the initial assumed postulate from which we start. We formulate the hypothesis that the information required for identifying at-risk profiles is available in the initial patients' drug prescription data at the time of hospital admission. In other terms, our system exclusively relies on analysing prescribed drug data of the day of admission. Previous studies that seeked to exploit EHR information with a similar postulate have mainly been developing score-based techniques. For instance the works found in $[33,34,35,5]$ also assume that the complexity of the patient's medication regimen is a good indicator of the complexity of the patient's condition. The existence of correlations between MRCI at admission and occurence of complications is empirically demonstrated in the work of Lepelley et al. [5]. Compared to these works, we go further by (1) exploring how this information can be leveraged for predictive purposes (on large datasets) and (2) by considering finer-grained features, thanks to the distributed architecture, which allows to improve prediction accuracy. A simple score such as MRCI constitutes a rough approximation. For example, the same MRCI value may denote different situations with radically different evolution perspectives. Our fine-grained approach is more adapted to capture these differences. We showed that this leads to increased prediction accuracy.

Last but not least, we provide experimental evidence to validate our claim that big data perspectives such as volume and variety effectively help in extracting relevant information useful in a novel and concrete healthcare application for predicting complications.

\section{Conclusion}

We propose a novel method for identifying patients at risk of complications during their hospital stay, which is based exclusively on drug prescription data of the day of admission, for the purpose of developing adapted prevention. We illustrate how the volume and variety perspectives of big medical data improve the automatic identification of at-risk patients. Experimental results suggest that such systems might be especially useful for detecting patient profiles at 
risk of hospital-acquired infections, pressure ulcers and death. This opens the door to promising research on the construction of further models. An advantage of our initial prototype system that of being adapted for deployment in hospitals as it is implementable in-house with modest hardware. It does not require neither external storage of sensitive medical data (thus avoiding additional issues of confidential data leakage), nor very expensive hardware (thanks to the distribution of data and computations).

\section{References}

[1] D. Levinson, Adverse Events in Hospitals: National Incidence among Medicare Beneficiaries, Tech. Rep., Department of Health and Human Services, USA, https://oig.hhs.gov/oei/reports/OEI-06-09-00090.pdf, 2010.

[2] C. Schaeffer, A. Haque, L. Booton, J. Halleck, A. Coustasse, Big Data Management in United States Hospitals: Benefits and Barriers., in: Proceedings of the Business and Health Administration Association Annual Conference, J. Sanchez (Ed.), 129-138, 2016.

[3] R. Hillestad, J. Bigelow, A. Bower, F. Girosi, R. Meili, R. Scoville, R. Taylor, Can Electronic Medical Record Systems Transform Health Care? Potential Health Benefits, Savings, And Costs, Health Affairs 24 (5) (2005) 1103-1117, doi:10.1377/hlthaff.24.5.1103, https://www .healthaffairs.org/doi/pdf/10.1377/hlthaff.24.5.1103.

[4] Frost, Sullivan, Drowning in Big Data? Reducing Information Technology Complexities and Costs For Healthcare Organizations, Tech. Rep., Frost \& Sullivan Company White Paper, 2012.

[5] M. Lepelley, C. Genty, A. Lecoanet, B. Allenet, P. Bedouch, M.R. Mallaret, P. Gillois, J.-L. Bosson, Electronic Medication Regimen Complexity Index at admission and complications during hospitalization in medical wards: a tool to improve quality of care?, International Journal for Quality in Health Care doi:10.1093/intqhc/mzx168, URL http://dx.doi.org/10.1093/intqhc/mzx168.

[6] H. Quan, V. Sundararajan, P. Halfon, A. Fong, B. Burnand, J.-C. Luthi, L. D. Saunders, C. Beck, T. Feasby, W. Ghali, Coding algorithms for defining comorbidities in ICD-9-CM and ICD-10 administrative data., Med Care 43 (11) (2005) 1130-1139, https://www.ncbi.nlm.nih.gov/pubmed/16224307.

[7] D. Roosan, M. Samore, M. Jones, Y. Livnat, J. Clutter, Big-Data Based Decision-Support Systems to Improve Clinicians' Cognition, in: Proceedings - 2016 IEEE International Conference on Healthcare Informatics, ICHI 2016, Institute of Electrical and Electronics Engineers Inc., 285-288, doi: 10.1109/ICHI.2016.39, 2016. 
[8] M. Armbrust, R. S. Xin, C. L., Y. H., D. L., J. K. Bradley, X. Meng, T. Kaftan, M. Franklin, A. Ghodsi, M. Zaharia, Spark SQL: Relational Data Processing in Spark, in: SIGMOD, 1383-1394, doi:10.1145/2723372.2742797, http://doi.acm.org/10.1145/2723372.2742797, 2015.

[9] Z. Czech, G. Havas, B. Majewski, Perfect Hashing, Theor. Comput. Sci. 182 (1-2) (1997) 1-143, doi:10.1016/S0304-3975(96)00146-6, http://dx.doi.org/10.1016/S0304-3975(96)00146-6.

[10] M. Zaharia, R. S. Xin, P. Wendell, T. Das, M. Armbrust, A. Dave, X. Meng, J. Rosen, S. Venkataraman, M. Franklin, A. Ghodsi, J. Gonzalez, S. Shenker, I. Stoica, Apache Spark: a unified engine for big data processing, Commun. ACM 59 (11) (2016) 56-65, doi:10.1145/2934664, URL http://doi.acm.org/10.1145/2934664.

[11] X. Meng, J. Bradley, B. Yavuz, E. Sparks, S. Venkataraman, D. Liu, J. Freeman, D. Tsai, M. Amde, S. Owen, D. Xin, R. Xin, M. Franklin, R. Zadeh, M. Zaharia, A. Talwalkar, MLlib: Machine Learning in Apache Spark, CoRR abs/1505.06807, http://arxiv.org/abs/1505.06807.

[12] D. Cox, The Regression Analysis of Binary Sequences, Journal of the Royal Statistical Society. Series B (Methodological) 20 (2) (1958) 215-242, ISSN 00359246, http://www.jstor.org/stable/2983890.

[13] J. Suykens, J. Vandewalle, Least Squares Support Vector Machine Classifiers, Neural Processing Letters 9 (3) (1999) 293-300, ISSN 1573-773X, doi:10.1023/A:1018628609742, URL http://dx.doi.org/10.1023/A:1018628609742.

[14] J. Quinlan, Induction of decision trees, Machine learning 1 (1) (1986) 81-106.

[15] J. Han, J. Pei, Y. Yin, R. Mao, Mining Frequent Patterns without Candidate Generation: A Frequent-Pattern Tree Approach, Data Min. Knowl. Discov. 8 (1) (2004) 53-87, doi:10.1023/B:DAMI.0000005258.31418.83, http://dx.doi.org/10.1023/B:DAMI.0000005258.31418.83.

[16] L. Maaten, G. Hinton, Visualizing data using t-SNE, Journal of Machine Learning Research 9 (Nov) (2008) 2579-2605.

[17] D. Merkel, Docker: Lightweight Linux Containers for Consistent Development and Deployment, Linux J. 2014 (239), ISSN 1075-3583, http://dl.acm.org/citation.cfm?id=2600239. 2600241.

[18] J. George, Y. Phun, M. J. Bailey, D. C. Kong, K. Stewart, Development and Validation of the Medication Regimen Complexity Index, Annals of Pharmacotherapy 38 (9) (2004) 1369-1376, doi:10.1345/aph.1D479, http://dx.doi.org/10.1345/aph.1D479. 
[19] A. Paquin, K. Zimmerman, T. Kostas, L. Pelletier, A. Hwang, M. Simone, L. Skarf, J. Rudolph, Complexity perplexity: a systematic review to describe the measurement of medication regimen complexity, Expert Opinion on Drug Safety 12 (6) (2013) 829-840, doi:10.1517/14740338.2013.823944.

[20] T. Fawcett, An Introduction to ROC Analysis, Pattern Recogn. Lett. 27 (8) (2006) 861-874, doi:10.1016/j.patrec.2005.10.010, http://dx.doi.org/10.1016/j.patrec.2005.10.010.

[21] D. Powers, Evaluation: From precision, recall and f-measure to roc., informedness, markedness \& correlation, Journal of Machine Learning Technologies 2 (1) (2011) 37-63.

[22] I. Guyon, V. Lemaire, M. Boullé, G. Dror, D. Vogel, Analysis of the KDD Cup 2009: Fast Scoring on a Large Orange Customer Database, in: Proc. of KDD-Cup competition, vol. 7 of JMLR Proceedings, 1-22, http://www.jmlr.org/proceedings/papers/v7/guyon09.html, 2009.

[23] J. Davis, M. Goadrich, The relationship between Precision-Recall and ROC curves, in: ICML, 233-240, doi:10.1145/1143844.1143874, http://doi.acm.org/10.1145/1143844.1143874, 2006.

[24] J. A. Pérez, C. C. Y. Poon, R. D. Merrifield, S. T. C. Wong, G. Yang, Big Data for Health, IEEE J. Biomedical and Health Informatics 19 (4) (2015) 1193-1208, doi:10.1109/JBHI.2015.2450362, https://doi.org/10.1109/JBHI . 2015.2450362.

[25] W. Lee, Y. Lee, H. Kim, I. Moon, Bayesian Nonparametric Collaborative Topic Poisson Factorization for Electronic Health Records-Based Phenotyping, in: IJCAI, 2544-2552, http://www.ijcai.org/Proceedings/16/Papers/362.pdf, 2016.

[26] Z. Kuang, J. A. Thomson, M. Caldwell, P. L. Peissig, R. M. Stewart, D. Page, Baseline Regularization for Computational Drug Repositioning with Longitudinal Observational Data, in: IJCAI, 2521-2528, http://www.ijcai.org/Proceedings/16/Papers/359.pdf, 2016.

[27] F. Li, Y. Zhang, M. Zhang, D. Ji, Joint Models for Extracting Adverse Drug Events from Biomedical Text, in: IJCAI 2016, 2838-2844, http://www.ijcai.org/Proceedings/16/Papers/403.pdf, 2016.

[28] Y. Luo, Y. Xin, R. Joshi, L. Celi, P. Szolovits, Predicting ICU Mortality Risk by Grouping Temporal Trends from a Multivariate Panel of Physiologic Measurements, in: AAAI, 42-50, https://www . aaai.org/ocs/index.php/AAAI/AAAI16/paper/view/11843/11562.pdf, 2016 .

[29] J. Zhang, L. Chen, A. Vanasse, J. Courteau, S. Wang, Survival Prediction by an Integrated Learning Criterion on Intermittently Varying Healthcare Data, in: AAAI, 72-78, 
http://aaai.org/ocs/index.php/AAAI/AAAI16/paper/view/11873/11566.pdf, 2016.

[30] A. Avati, K. Jung, S. Harman, L. Downing, A. Y. Ng, N. H. Shah, Improving Palliative Care with Deep Learning, in: Proceedings of the IEEE International Conference on Bioinformatics and Biomedicine, 311-316, https://arxiv.org/pdf/1711.06402.pdf, 2017.

[31] A. Rajkomar, E. Oren, K. Chen, A. M. Dai, N. Hajaj, P. J. Liu, X. Liu, M. Sun, P. Sundberg, H. Yee, K. Zhang, G. E. Duggan, G. Flores, M. Hardt, J. Irvine, Q. Le, K. Litsch, J. Marcus, A. Mossin, J. Tansuwan, D. Wang, J. Wexler, J. Wilson, D. Ludwig, S. L. Volchenboum, K. Chou, M. Pearson, S. Madabushi, N. H. Shah, A. J. Butte, M. Howell, C. Cui, G. Corrado, J. Dean, Scalable and accurate deep learning for electronic health records, Tech. Rep., eprint arXiv:1801.07860: https://arxiv.org/abs/1801.07860, 2018.

[32] R. Caruana, Y. Lou, J. Gehrke, P. Koch, M. Sturm, N. Elhadad, Intelligible Models for HealthCare: Predicting Pneumonia Risk and Hospital 30-day Readmission, in: Proceedings of the 21th ACM SIGKDD International Conference on Knowledge Discovery and Data Mining, Sydney, NSW, Australia, August 10-13, 2015, 1721-1730, doi:10.1145/2783258.2788613, http://doi.acm.org/10.1145/2783258.2788613, 2015.

[33] H. Schoonover, C. Corbett, D. Weeks, M. Willson, S. Setter., Predicting potential postdischarge adverse drug events and 30-day unplanned hospital readmissions from medication regimen complexity., J Patient Saf. 10 (4) (2014) 186-91, doi:10.1097/PTS.0000000000000067, https://www.ncbi.nlm.nih.gov/pubmed/25408236.

[34] M. Willson, CL.Greer, D. Weeks., Medication regimen complexity and hospital readmission for an adverse drug event., Ann Pharmacother. 48 (1) (2014) 26-32, doi:10.1177/1060028013510898., https://www.ncbi.nlm.nih.gov/pubmed/24259639.

[35] F. Yam, T. Lew, S. Eraly, H. Lin, J. Hirsch, M. Devor, Changes in medication regimen complexity and the risk for 90-day hospital readmission and/or emergency department visits in U.S. Veterans with heart failure., Res Social Adm Pharm. 12 (5) (2016) 713-21, doi:10.1016/j.sapharm.2015.10.004., https://www.ncbi.nlm.nih.gov/pubmed/26621388. 